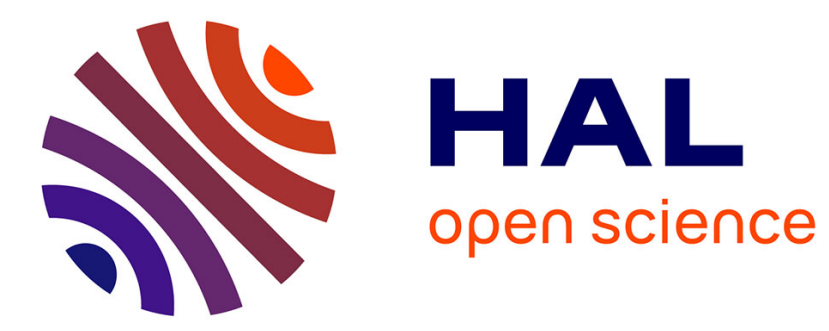

\title{
Quasi resonant collisional excitation transfer between Zeeman sublevels in high magnetic fields

\author{
J.-C. Gay, W.B. Schneider
}

\section{To cite this version:}

J.-C. Gay, W.B. Schneider. Quasi resonant collisional excitation transfer between Zeeman sublevels in high magnetic fields. Journal de Physique Lettres, 1975, 36 (7-8), pp.185-187. 10.1051/jphyslet:01975003607-8018500 . jpa-00231184

\section{HAL Id: jpa-00231184 https://hal.science/jpa-00231184}

Submitted on 1 Jan 1975

HAL is a multi-disciplinary open access archive for the deposit and dissemination of scientific research documents, whether they are published or not. The documents may come from teaching and research institutions in France or abroad, or from public or private research centers.
L'archive ouverte pluridisciplinaire HAL, est destinée au dépôt et à la diffusion de documents scientifiques de niveau recherche, publiés ou non, émanant des établissements d'enseignement et de recherche français ou étrangers, des laboratoires publics ou privés. 


\title{
QUASI RESONANT COLLISIONAL EXCITATION TRANSFER BETWEEN ZEEMAN SUBLEVELS IN HIGH MAGNETIC FIELDS
}

\author{
J.-C. GAY and W. B. SCHNEIDER $\left(^{*}\right)$
}

Laboratoire de Spectroscopie Hertzienne de l'ENS (**) et Université Paris VII

4, place Jussieu, Tour 12, 1er étage, 75230 Paris Cedex 05, France

(Reçu le 4 avril 1975, accepté le 6 mai 1975)

\begin{abstract}
Résumé. - La décroissance du taux de transfert entre sous-niveaux Zeeman du niveau $6{ }^{3} P_{1}$ en champ magnétique intense est étudiée dans le cas des collisions $\mathrm{Hg}^{*}$ - $\mathrm{Hg}$ et comparée à la théorie. Les résultats de l'étude préliminaire dans une gamme de champ de 8 teslas des collisions $\mathbf{H g}$ *-gaz rares sont également donnés.
\end{abstract}

Abstract. - Experimental evidence of the decrease of the $\mathrm{Hg}-\mathrm{Hg}$ * collisional transfer rate between Zeeman sublevels of the $6{ }^{3} P_{1}$ state with varying magnetic fields (up to $80 \mathrm{kG}$ ) is reported and compared with theory. Preliminary results on $\mathrm{Hg}^{*}$-rare gas collisions are also given for a field of $80 \mathrm{kG}$.

1. Introduction. - The excitation transfer between levels of atoms or molecules by collisions at thermal energies is of great interest especially when it is possible to vary the energy difference between these levels. Unfortunately, in most of the experimental situations it is not possible to achieve this variation without changing the atomic species under consideration (e. g. alkali atoms and transfer between fine structure levels) and under these conditions, the potential energy also changes. A powerful method permitting a continuous variation of the energy difference between the levels can be achieved by using magnetic fields and studying the collisional transfer between Zeeman sublevels. This transfer becomes weaker when the Zeeman splitting of the levels $\Delta E$ is greater than $\hbar$ divided by $\tau_{\mathrm{c}}$ the mean duration of the collision. This suggests the definition of a critical value for the field, such that $\Delta E . \tau_{\mathrm{c}} \approx \hbar$, which roughly characterizes the decrease of the transfer rates with the field. This critical field value is about $10 \mathrm{kG}$ for $\mathrm{Hg}^{*}-\mathrm{Hg}$ resonant collisions in the $6{ }^{3} \mathrm{P}_{1}$ state and about $30 \mathrm{kG}$ for $\mathrm{Hg}^{*} \mathrm{Xe}$ non resonant collisions $\left({ }^{1}\right)$. In the following, we

(*) On leave from Fachbereich Physik, Universität Marburg/L. (**) Laboratoire associé $\mathrm{n}^{0} 18$

(1) The critical field value is defined with $\tau_{\mathrm{c}}=\sqrt{\frac{\sigma}{\pi}} v^{-1}$ where $\sigma$ is the cross section and $v$ the mean relative velocity. But fields several times greater than the critical field as defined above are needed to observe significant variations of the transfer rates. report the experimental study of $\mathrm{Hg}^{*}$ - $\mathrm{Hg}$ collisions [1] and preliminary results on $\mathrm{Hg}^{*}$-rare gas collisions.

2. Experimental. - The experiments are performed on the $6{ }^{3} \mathrm{P}_{1}$ state of ${ }^{198} \mathrm{Hg}$ (purity better than $99.7 \%$ ). The resonance cell filled with this isotope is placed at the center of a superconductive magnet capable of producing fields up to 8 teslas. $\mathrm{A}^{198} \mathrm{Hg} \mathrm{R}$. F. discharge lamp in zero field is used to excite the $m=0$ Zeeman sublevel which is not shifted by the field. The $\sigma$ and $\pi$ fluorescence intensities are detected at right angles to the excitation direction (Fig. 1). The polarizers are placed close to the cell in order to suppress depolariza-

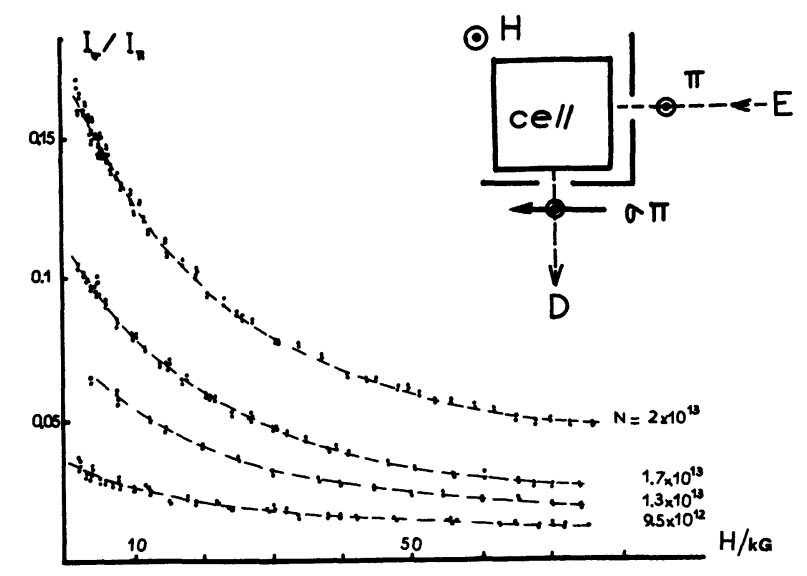

Fig. 1. - Geometrical arrangement of the beams and variations with the field of $I_{\sigma} / I_{\pi}$ at various mercury densities $\left(N\right.$ in $\left.\mathrm{cm}^{-3}\right)$. 
tion effects by optics (mirrors, lenses and flexible light guides). Furthermore, slits are used to reduce the angular aperture of the beams and the directly detected excitation light. The $\sigma$ and $\pi$ fluorescence intensities are detected by means of a magnetic field-shielded photomultiplier and measured with a digital voltmeter. The vapour pressure in the cell is determined by the temperature of its side arm. During measurements the stability of the lamp is monitored with a photocell.

We can insert various mercury absorption chambers filled with pure isotopes (purity better than $99.6 \%$ ) into the excitation and the detection beams. $\mathrm{A}{ }^{198} \mathrm{Hg}$ filter is used to absorb the small amount of $\pi$ light which is mixed with the $\sigma$ light because of the imperfections of the detection polarizer. We have also verified that there is no direct excitation of the $\sigma$ components of ${ }^{198} \mathrm{Hg}$ in the cell by isotopes at low concentration in the lamp or by a possible continuous spectra emitted by it. So the $\sigma$ signal we record is really due to the collisional transfer between the $m=0$ and $m= \pm 1$ Zeeman sublevels of the atoms contained in the cell.

3. Theoretical interpretation of the signals. - The stationary solution of the rate equation for the populations of the $m= \pm 1\left(\rho_{\sigma}\right)$ and $m=0\left(\rho_{\pi}\right)$ Zeeman sublevels is given by :

$$
\dot{\rho}_{\sigma}=0=-\left(\Gamma^{\sigma}+g^{\sigma}+g\right) \rho_{\sigma}+\left(g^{\sigma}+g\right) \rho_{\pi}
$$

$\Gamma^{\sigma}$ represents the inverse of the imprisonment time of the $\sigma$ photons in the cell, $g^{\sigma}$ the transfer rate between the $m=0$ and $m= \pm 1$ sublevels due to $\mathrm{Hg}^{*}-\mathrm{Hg}$ resonant collisions linearly depending on the mercury atom density $N$ and $g$ the transfer rate due to non resonant $\mathrm{Hg}^{*}$-rare gas collisions (depending on rare gas pressure).

Hence the stationary values of $\rho_{\sigma}$ and $\rho_{\pi}$ are such that :

$$
\frac{\rho_{\sigma}}{\rho_{\pi}}=\frac{g^{\sigma}+g}{\Gamma^{\sigma}+g^{\sigma}+g} .
$$

In first approximation we assume that the ratio $I_{\sigma} / I_{\pi}$ of the $\sigma$ and $\pi$ fluorescence light can be expressed [2]

$$
\rho=\frac{I_{\sigma}}{I_{\pi}}=\lambda(N) \frac{\rho_{\sigma}}{\rho_{\pi}}
$$

where $\lambda(N)$ depends on the width of the excitation profile, mercury density, the spatial excitation distribution in the cell, and the sizes of the slits and the cell. $\lambda(N)$ does not depend on $H$ in our experimental conditions.

As multiple scattering is a non-local process, the expressions (1) and (2) involve several approximations but they are justified in the density range in which the experiments were performed.
4. Variations of the collisional transfer rate with the field in $\mathbf{H g}$ *-Hg resonant collisions. - The experiments are performed under the conditions $\Gamma^{\sigma} \gg g^{\sigma}$ (mercury density between $10^{13}$ and $2 \times 10^{13} \mathrm{~cm}^{-3}$ ) and $g \simeq 0$ (pure ${ }^{198} \mathrm{Hg}$ filled cells). Eq. (2) becomes

$$
\rho \simeq \frac{\hat{\lambda}(N)}{\Gamma^{\sigma}(N)} g^{\sigma} .
$$

The ratio of the intensities is therefore proportional to the collisional transfer rate $g^{\sigma}$ between the $m=0$ and $m= \pm 1$ Zeeman sublevels and to $\lambda(N) / \Gamma^{\sigma}(N)$ which depends on $N$ because of the multiple scattering process.

The measured field dependence of the ratio $I_{\sigma} / I_{\pi}$ at various mercury densities, is given on figure 1 and reveals a very important decrease of $\rho$ with the field. This is due to the decrease of the collisional transfer rate $g^{\sigma}$ as the energy difference between the levels is increased.

This has been carefully verified since it is possible to imagine some other explanation connected with the lack of homogeneity of the field. If indeed the variations of the field between two successive absorptions of a photon in two different parts of the cell are of the order of magnitude of the Doppler width, then the probability of reabsorption of the photon will be decreased. This is clearly impossible for $\pi$ but not for $\sigma$ photons and the imprisonment time of the $\sigma$ photons may decrease as the field is increased. Such an interpretation has been invalidated using several methods which will be reported later [3]. But such an effect can impose severe restrictions in higher fields or worse homogeneity conditions [3].

In order to compare the experimental variations of $g^{\sigma}$ with theoretical investigations [4], we plot in figure 2 the values of $\rho(H) / \rho(5)$ against the field at

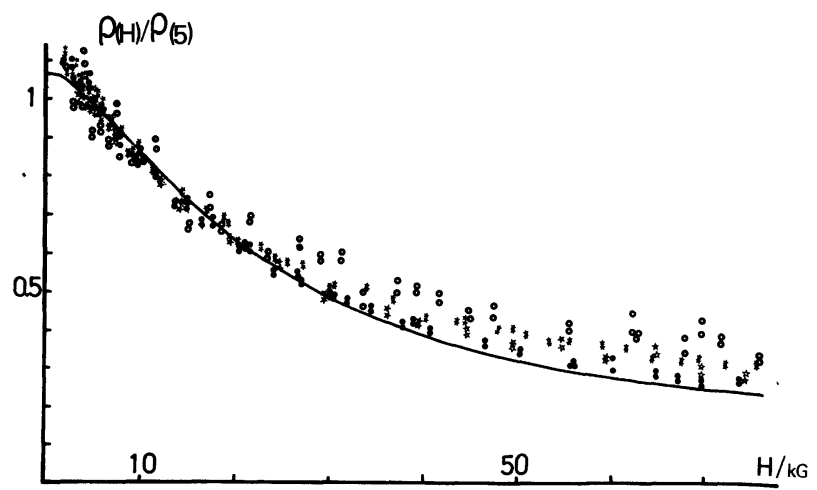

FIG. 2. - Variations with the field of $\rho(H) / \rho(5)(H$ in $\mathrm{kG})$

- $N=1.7 \times 10^{13} \mathrm{~cm}^{-3} \nless \mathrm{V}=2.05 \times 10^{13} \mathrm{~cm}^{-3}$

$\$ N=1.26 \times 10^{13} \mathrm{~cm}^{-i} \mathrm{~V}=9.5 \times 10^{12} \mathrm{~cm}^{-3}$

- - Theory (mean velocity assumed)

Theory (velocity averaging)

various mercury densities $(\rho(5)$ is the value taken at $H=5 \mathrm{kG}$ ). It is not possible to use smaller values of the field to normalize the results because of : 
a) the change in multiple scattering when the Zeeman splitting is smaller than the Doppler width (the $\sigma$ and $\pi$ photons are no longer diffused independently in these conditions, formula (2) breaks down and the experimental results for $H \lesssim 2 \mathrm{kG}$ cannot be compared with those obtained in higher fields) ;

b) the necessity to avoid all the effects connected with direct excitation of the $m= \pm 1$ levels by photons belonging to the wings of the excitation profile, or possible anomalous dispersion effects. Such effects possibly cause the slightly high values of $\rho(H) / \rho(5)$ between 1 and $3 \mathrm{kG}$.

As $\rho(H) / \rho(5)$ is just $g^{\sigma}(H) / g^{\sigma}(5)$ (eq. (3)), it does not theoretically depend on $N$ which seems well verified in figure 2 within the experimental uncertainties. Nevertheless, a slight discrepancy appears in high fields for the smallest value of the density. This is due to a small amount of residual gas in the cell, the effect of which is to create an additional transfer between the Zeeman sublevels of mercury (eq. (2)). As such a process does not to a good approximation depend on the field strength, the relative contribution to the transfer of $\mathrm{Hg}^{*}$-residual gases collisions will increase with the field, and will no longer be negligible at low mercury densities. Such an effect is almost negligible for densities of about $1.5 \times 10^{13} / \mathrm{cm}^{3}$ and greater.

The theoretical curves are shown in figure 2 with and without velocity averaging. The hypothesis and outline of the calculations [4] are very similar to those of a previous paper [5]. The fundamental assumption still remains that $R^{-3}$ dipole-dipole interaction is responsible for the transfer, but the relaxation matrix obtained in reference [4] does not have spherical symmetry in Liouville space. The agreement between theory and experiment will be discussed extensively in reference [4]. It seems to confirm, for a large range of applied magnetic field, the validity of the dipole-dipole interaction invoked to account for collisional phenomena between two identical mercury atoms, one of them in the ground $6{ }^{1} \mathrm{~S}_{0}$ state and the other one in the $6{ }^{3} \mathrm{P}_{1}$ state. Similar results are obtained for $\mathrm{Na}^{*}-\mathrm{Na}$ collisions and will be reported later [6].

During the course of this work, reference [7] by Fuchs et al. was published and the interpretation of the results quoted therein warrants some remarks. Firstly the authors use mercury of insufficient isotopic purity which makes it difficult to arrive at a simple interpretation of the results in the range $0-10 \mathrm{kG}$. Secondly, the experimental conditions mentionned on figure 2 of reference [7] (Xe pressure of 0.75 torr, side arm temperature of $0^{\circ} \mathrm{C}$ ) are such that the transfer between Zeeman sublevels resulting from $\mathrm{Hg}^{*}-\mathrm{Hg}$ collisions is about $10^{-3}$ time smaller than the one due to $\mathrm{Hg}^{*}-\mathrm{Xe}$ collisions. It therefore seems hardly possible that $\mathrm{Hg}^{*}-\mathrm{Hg}$ collisions are responsible for the variations between 0-3 $\mathrm{T}$ reported in reference [6].

5. Mercury-rare gases collisions in high fields. The experiments are performed with cells containing various rare gases at different pressures, and at low mercury densities $\left(10^{11} \mathrm{at} / \mathrm{cm}^{3}\right)$. The $\mathrm{Hg} *-\mathrm{Hg}$ resonant collisions are therefore negligible and multiple scattering of the resonance line is very weak. $\Gamma^{\sigma}$ is reduced to $\Gamma$ (natural width of the $6{ }^{3} \mathrm{P}_{1}$ state), and $\lambda$ is equal to 1. Formula (2) becomes

$$
\rho=\frac{I_{\sigma}}{I_{\pi}}=\frac{g}{\Gamma+g} .
$$

For such non resonant collisions, the critical field is between $30 \mathrm{kG}$ and $300 \mathrm{kG}$, depending on the perturber.

The relative variations of the transfer rate between 0 and $60 \mathrm{kG}$ are plotted in figure 3 as a function of the inverse of the critical field under various experimental conditions. In spite of the dispersion of the measurements figure 3 shows that there is a variation when the

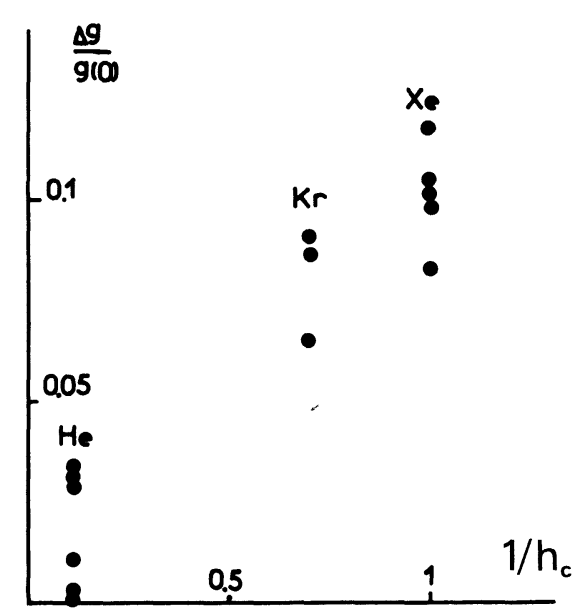

Fig. 3. - Relative variations $(\Delta g=g(0)-g(60))$ of the collisional transfer rates between 0 and $60 \mathrm{kG}$ as a function of the inverse of the critical field $h_{\mathrm{c}}$ (reduced to that of Xenon).

perturber is $\mathrm{Xe}$ or $\mathrm{Kr}$ but that there is no significant effect for Helium. (The critical field for the latter is about $300 \mathrm{kG}$.) Higher values of the field are of course necessary to complete these results.

\section{References}

[1] Gay, J.-C., Schneider, W. and Omont, A., Fourth I. C. A. P. (Heidelberg) July 1974.

[2] Omont, A., Thèse de $3^{\text {e }}$ cycle (Paris 1961) unpublished. Omont, A. and Brossel, J., C. R. Hebd. Séan. Acad. Sci. 252 (1961) 710.

Omont, A., C. R. Hebd. Séan. Acad. Sci. 252 (1961) 861.

[3] Gay, J.-C., Schneider, W. and OMONT, A., to be published in $J$. Physique.
[4] Gay, J.-C., to be published in J. Physique.

[5] GAY, J.-C., J. Physique 35 (1974) 813.

[6] SChNeIder, W., GAY, J.-C. and OMONT, A., to be published in J. Physique.

[7] Fuchs, B., Hanle, W., Oberheim, W. and Scharmann, A., Phys. Lett. 50 (1974) 337. 